\title{
Competitive dynamics in two species of marine phytoplankton under non-equilibrium conditions
}

\author{
Pedro Cermeño ${ }^{1,4, *}$, Joon-Baek Lee ${ }^{1,2}$, Kevin Wyman $^{1}$, Oscar Schofield ${ }^{1}$, \\ Paul G. Falkowski ${ }^{1,3}$ \\ ${ }^{1}$ Environmental Biophysics and Molecular Ecology Program, Institute of Marine and Coastal Sciences, Rutgers University, \\ 71 Dudley Road, New Brunswick, New Jersey 08901, USA \\ ${ }^{2}$ Department of Earth and Marine Sciences, College of Ocean Sciences, and Marine and Environmental Research Institute, \\ Jeju National University, Jeju 690-756, ROK \\ ${ }^{3}$ Department of Earth and Planetary Sciences, Rutgers University, 610 Taylor Road, Piscataway, New Jersey, 08854, USA \\ ${ }^{4}$ Present address: Departamento de Ecología y Biología Animal, Universidad de Vigo, 36310 Vigo, Spain
}

\begin{abstract}
Although mathematical models suggest that competition between primary producers in response to dynamical changes in the availability of a limiting nutrient is non-linear, experimental data supporting this basic hypothesis are sparse. Using continuous culture systems with nitrate as a single limiting nutrient, we present results of competition experiments between 2 species of marine phytoplankton, a diatom Thalassiosira pseudonana and a coccolithophore Coccolithus braarudii. These 2 organisms of similar size represent biogeochemically and ecologically distinct functional groups. Consistent with classical resource competition theory, under steady-state nitrate limitation (i.e. continuous flow chemostats), the diatom was outcompeted by the coccolithophore. However, when pulses of nitrate were provided to the chemostats (i.e. non-equilibrium, dynamical conditions) the diatom outcompeted the coccolithophore. The rate of exclusion was a linear function of the frequency of nitrate pulses. These results experimentally demonstrate that dynamical nutrient supply allows co-existence of 2 primary producers competing for a single limiting nutrient and may help us to understand phytoplankton succession in the ocean.
\end{abstract}

KEY WORDS: Ecological succession · Marine phytoplankton · Competition · Nutrient perturbation · Domain shift $\cdot$ Ocean turbulence $\cdot$ Climate change

\section{INTRODUCTION}

Diatoms and coccolithophorids largely dominate the biologically-mediated, net exchange of carbon dioxide between the atmosphere and the ocean (Smetacek 1999, Falkowski 2002). Owing to their large range of cell sizes and mineral skeletons, these 2 phytoplankton functional groups (sensu Falkowski et al. 2003) are responsible for a large fraction of export flux and burial of biogenic carbon in the ocean interior; i.e. the so-called 'biological pump' (Volk \& Hoffert 1985, Smetacek 1999, Iglesias-Rodríguez et al. 2002). However, their roles in the carbon cycle are strikingly different. Diatoms constitute a classic sink for carbon dioxide through the process of photosynthesis. However, by precipitating calcium carbonate, coccolithophorids decrease the alkalinity of seawater, release carbon dioxide, and hence, decrease the efficiency of the biological pump (Holligan \& Robertson 1996, Treguer \& Pondaven 2000). This biogeochemical control may have contributed significantly to the regulation of Earth's climate, probably since the beginning of the Cenozoic ( 65 million years ago) (Holligan \& Robertson 1996, Falkowski 2004). Understanding the mechanisms that govern the assembly and temporal distribution patterns of these 2 phytoplankton functional groups has increasingly become the focus of marine microbial ecologists and biogeochemists (Archer et al. 2000, Boyd \& Doney 2002; Hood et al. 2006).

Nutrients can control phytoplankton community composition through interspecific competition regulated 
either by (1) the compositional ratio or stoichiometry of nutrients (the so-called 'resource ratio hypothesis') or (2) the kinetics of nutrient input and uptake (the socalled 'dynamical nutrient supply hypothesis'). For instance, the correlation between the classical springsummer succession of phytoplankton in the coastal ocean and the silica to nitrate supply ratio has been well documented, initially favoring diatoms when silica is replete and other non-siliceous phytoplankton taxa such as coccolithophores and small flagellates later when silica becomes depleted (Smayda 1989, Sieracki et al. 1993). The resource-ratio hypothesis predicts the replacement and/or coexistence of species as a function of the ratio of limiting nutrients and has been well demonstrated under laboratory controlled conditions and in field experiments (Tilman 1982, Tilman et al. 1982, Sommer 1983, Grover 1989, Egge \& Aksnes 1992, Sommer 1993, Miller et al. 2005). In contrast, the dynamical nutrient supply hypothesis, which postulates that the timing of delivery of nutrients provides an ecological selection pressure, has received far less experimental support (Sommer 1985, Grover 1991a, Ducobu et al. 1998).

The role of nutrient supply dynamics was proposed originally by Hutchinson (1961, p. 137) to potentially explain the 'paradox of the plankton' in which he posed, 'The problem that is presented by the phytoplankton is essentially how it is possible for a number of species to coexist in a relatively isotropic or unstructured environment all competing for the same sorts of materials.' Subsequently, Ramon Margalef in his (now famous) 'mandala' suggested that different combinations of nutrients and turbulence select for phytoplankton taxa according to species-specific functional traits and survival strategies (Margalef 1978, Smayda \& Reynolds 2001). Based on the conceptual foundations of the 'mandala', resource-based competition models suggested that, owing to the rapid uptake of nutrients by diatoms, increased ocean turbulence (i.e. higher frequency of nutrient 'pulses') potentially would increase their ecological and evolutionary success at the expense of slow-growing coccolithophorids (Tozzi et al. 2004). Consistent with these theoretical predictions, we found that the onset or breakdown of stratification dramatically increases or decreases, respectively, the coccolithophorid to diatom biomass ratio in historical data sets from the ocean (Cermeño et al. 2008). Thus, both models and empirical evidence led to the hypothesis that enhanced ocean turbulence increases the rate of taxonomic shift from coccolithophore- to diatomdominated assemblages (and vice versa) with important implications for understanding community dynamics and ocean biogeochemistry (Tozzi et al. 2004, Falkowski \& Oliver 2007, Cermeño et al. 2008). Here, we explore the dynamical nutrient supply hypothesis in a laboratory-simulated microcosm containing a diatom and a coccolithophore.

\section{Competition for a single limiting nutrient: steady- state vs. non-steady-state nitrate inputs}

Classical competition theory dictates that the success of an individual phytoplankton taxa reflects the intracellular nutrient quota and uptake rate where the organism with the lower requirement for the limiting nutrient is at a competitive advantage (Stewart \& Levin 1973, Tilman 1977, Grover 1990). Several physiological traits characterize nutrient uptake kinetics and population growth, and their values directly affect the performance and competitive abilities of phytoplankton taxa.

The growth rate $(\mu)$ of a species is a saturating function of cell quota $\left(Q, \mu\right.$ mol nitrate cell $\left.{ }^{-1}\right)$ :

$$
\mu=\mu_{\max }\left(\frac{1-\frac{Q_{\min }}{Q}}{1-\frac{Q_{\min }}{Q_{\max }}}\right)
$$

As growth rate approaches zero, $Q$ approaches a non-zero, minimum quota $\left(Q_{\min }\right)$, and when growth asymptotically approaches a maximum, $Q$ approaches its maximum potential $\left(Q_{\max }\right)$. This 'Droop' model assumes that algal yield (i.e. the efficiency of algae to convert nutrients into biomass) depends on the physiological state of the population, which is primarily determined by cell quotas (Droop 1973, Grover 1991b).

The rate of change of population abundance $(N)$ is affected by the growth rate of the population and its mortality rate $(m)$ :

$$
\frac{\mathrm{d} N}{\mathrm{~d} t}=\mu(Q) N-m N
$$

Nutrient uptake rate $\left(\rho, \mu \mathrm{mol}\right.$ nutrient cell $\left.{ }^{-1} \mathrm{~d}^{-1}\right)$ is an increasing function of external nutrient concentration $\left(R, \mu \mathrm{mol} \mathrm{l}^{-1}\right)$ and can be parameterized by a hyperbolic function that is constrained by a maximum nutrient uptake rate $\left(\rho_{\max }\right)$ and half saturation constant $(K, \mu$ mol $\mathrm{l}^{-1}$ ) for the limiting nutrient:

$$
\rho=\left(\frac{\rho_{\max } R}{K+R}\right)
$$

If the nutrient concentration in the bulk media is elevated to levels that are saturating for the uptake system, then the maximal growth rate becomes constrained by an upper bound of $Q$. Under these circumstances, the maximum growth rate depends on the efficiency of the photosynthetic machinery to convert nutrients into biomass with a fixed elemental stoichiometry (Falkowski \& Raven 1997). 
The rate of change of $Q$ is dependent upon the nutrient uptake rate, the maximal growth rate and the difference between the instantaneous cell quota and the minimal cell quota. The larger this difference, the slower the rate of change of the cell quota:

$$
\frac{\mathrm{d} Q}{\mathrm{~d} t}=\rho-\mu_{\max }\left(Q-Q_{\min }\right)
$$

The rate of change of $R$ is a function of the dilution rate $(D)$ and the total uptake by the organisms:

$$
\frac{\mathrm{d} R}{\mathrm{~d} t}=D\left(R^{0}-R\right)-N \rho
$$

where $\mathrm{R}^{0}$ is the initial nutrient concentration entering the system.

According to this set of equations, the speciesspecific nutrient requirement at equilibrium $\left(R^{*}\right)$ can be calculated for each species growing alone and in the absence of competitors as:

$$
R^{*}=\frac{K \mu_{\max } Q_{\max } Q_{\min } m}{\mu_{\max } Q_{\max }\left(\rho_{\max }-Q_{\min } m\right)-\rho_{\max } m\left(Q_{\max }-Q_{\min }\right)}
$$

such that a species with the lowest $R^{*}$ is predicted to win in a competition for a given single limiting nutrient (Tilman 1977, Grover 1991b). Following this logic, Tilman (1977) demonstrated that the outcome of competition for silicate among planktonic diatoms closely follows the $R^{*}$ rule at steady state. However, the prediction given by $R^{*}$ may change if the system does not reach equilibrium, as in the case of variable resource supply regimes (Grover 1991b, Miller et al. 2005).

High maximum nutrient uptake rates (i.e. 'luxury' uptake) and storage capabilities by diatoms are advantageous physiological traits under non-equilibrium conditions (Grover 1988, Legovic \& Cruzado 1997, Litchman et al. 2007). Intracellular storage capacity introduces a time lag between the exhaustion of the external nutrient concentration and the actual nutrient limitation of growth, thereby extending the range of environmental conditions and allowing for maximal growth (Droop 1973, Grover 1991b). Furthermore, by confining acquired nutrients to a storage vacuole, cells potentially enhance the nutrient gradient across the plasmalema (Raven 1997).

When a single limiting nutrient is temporally variable, the outcome of competition is controlled by temporal changes in the nature and strength of competitive interactions. In the steady state, continuous nutrient limitation is predicted to favor coccolithophores by virtue of their lower half-saturation constants for nutrient uptake and small intracellular quotas (Tozzi et al. 2004). Conversely, intermittent (non-equilibrium) nutrient supply potentially allows diatoms with higher maximum uptake rates to con- sume and store the bulk of the nutrients rapidly, thereby permitting them to attain higher growth rates for several generations, while denying the coccolithophore access to the limiting resource (Tozzi et al. 2004, Litchman et al. 2007). The effect on external nutrient concentrations is even more dramatic, taking into account that fast growing cells possess high nutritional requirements (i.e. low algal yield) (Aksnes \& Egge 1991), further destabilizing the competitive equilibrium towards the exclusion of slow-growing species.

To test this hypothesis, we designed a suite of experiments in which competition was based on a single limiting nutrient, nitrate, which is required by both the diatom and the coccolithophore. Here, we present the response of these 2 phytoplankton in laboratorysimulated dynamic ecosystems in which the limiting nutrient is provided as pulses at different frequencies. The experimental results clearly demonstrate that coexistence of these 2 species, each competing for the same limiting nutrient, can be maintained under dynamic conditions but not under continuous nutrient limitation.

\section{MATERIALS AND METHODS}

The 2 strains used, the diatom Thalassiosira pseudonana (CCMP-1335) and the coccolithophore Coccolithus braarudii (RCC-1201; Roscoff), have relatively similar cell volumes, averaging 180 and $520 \mathrm{\mu m}^{3}$, respectively, thereby minimizing allometric effects on metabolic rates. Cultures were grown in monospecific continuous culture systems using 11 temperature controlled culture vessels with nitrate limited-f/2 + Si medium using pre-filtered and autoclaved natural seawater. Nitrate limitation was obtained by diluting the nitrate stock 10 -fold, thereby giving a final concentration in the medium of $88.2 \mu \mathrm{mol} \mathrm{l}^{-1}$ and a N/P molar ratio of $\sim 2.5$ for the inflow media. The final nutrient concentrations of $\mathrm{N}, \mathrm{P}$ and $\mathrm{Si}$ in the medium were 88.2, 36.2 and $106 \mu \mathrm{mol} \mathrm{l}^{-1}$, respectively. High-precision metering pumps (Q pumps, Fluid Metering) delivered media to the culture vessel with a balancing overflow to maintain a constant volume in the chemostats. In the steady state, the growth rate of the cells is equal to the dilution rate. The dilution rate was set to $0.25 \mathrm{~d}^{-1}$, which is in the range of average growth rates experienced by field populations of algae in oligotrophic, near steady-state ecosystems, and represents the mortality rate in the theoretical model above (i.e. the dilution rate of our continuous culture systems). The cultures were illuminated continuously ( $24 \mathrm{~h})$ by a bank of $8 \mathrm{cool}$ white plus fluorescent lamps (30W, Phillips,) emitting a photon flux of $\sim 200 \mu \mathrm{mol}$ quanta $\mathrm{m}^{-2} \mathrm{~s}^{-1}$. We used a continuous light regime instead of a light:dark 
cycle, as the progressive accumulation of nutrients overnight would bias the competitive interactions in favor of the faster growing diatom (Stolte \& Riegman 1995). Temperature in the cultures was maintained at $18 \pm 1^{\circ} \mathrm{C}$ by circulating water through the vessel jackets using a temperature controlled water bath. The cultures were stirred and aerated through $0.2 \mu \mathrm{m}$ filters throughout the experiments with magnetic stirrers and air pumps. The culture systems, from reservoirs to outflow flasks, were sterilized by autoclaving. Before starting nutrient uptake kinetics and competition experiments, independent monospecific cultures were grown for at least $1 \mathrm{wk}$ in nitrate-limited continuous cultures.

Maximum nutrient uptake rates and half saturation constants were determined on monospecific cultures grown at steady-state. The nitrate concentration in the bulk media was measured at different time intervals in a series of aliquots to which different concentrations of nitrate were initially added $(0.25,0.5,1,2.5,5,10$, $25 \mu \mathrm{mol} \mathrm{\textrm {l } ^ { - 1 }}$ ). Nitrate concentrations were measured colorimetrically on seawater samples filtered through $0.45 \mu \mathrm{m}$ pore size polycarbonate syringe filters, following the methods described in Grasshoff (1976). Cellspecific maximum nitrate uptake rates and half saturation constants were then calculated by fitting a hyperbolic function to the data. Data correspond to 2 different sets of independent measurements from which a single nitrate uptake kinetic curve was generated for each population.

Competition for nitrate between the diatom Thalassiosira pseudonana and the coccolithophore Coccolithus braarudii was simulated in mixed-population continuous culture systems. The culture conditions were similar to those specified above for monospecific cultures with the exception that the inflow media was supplemented with discrete pulses of nitrate $(7 \mu \mathrm{mol}$ $\left.\mathrm{l}^{-1}\right)$ from a sterile stock solution $\left(883 \mu \mathrm{mol} \mathrm{l^{-1 }}\right)$ at predetermined intervals $\left(0.5,1,2\right.$ and 5 pulses $\left.\mathrm{d}^{-1}\right)$. The experimental matrix physically consisted of a nutrient reservoir connected to 3 digitally controlled solenoid valves where nutrients were directed to each of 3 culture vessels operating in parallel under identical light and temperature conditions. The nitrate pulses were added with a pipette through a septum in the top of the growth chamber. This procedure allowed us to rapidly increase the nitrate concentration in the bulk media without materially altering the dilution rate of the system; a total $8 \mathrm{ml}$ of stock solution was added to the growth chamber in each pulse, which amounted to a $0.8 \%$ change in culture volume.

In the competition experiments, the initial phase was set up as a continuous culture system that was shifted to a pulsed regime in the second phase of the experiments. In all of the experiments, silica availability exceeded the growth demands of the diatom, and trace metals were not limiting. The cells were counted and sized daily using a Multisizer III Coulter Counter (Beckman Coulter). Population densities and cell size on fresh samples were further verified by quantitative microscopy using an inverted microscope (Utermöhl's method). Key photosynthetic parameters $\left(F_{\mathrm{v}} / F_{\mathrm{m}}\right.$ : variable/maximum fluorescence; $\sigma_{\text {PSII }}$ : absorption cross section of photosystem $\mathrm{II}_{;} \tau$ : turnover rate of photosystem II) indicative of the photosynthetic energy conversion efficiency of the entire assemblage were determined using a custom-built FiRE Fluorometer (Gorbunov et al. 1999).

In theory, the outcome of competition is independent of the initial population densities. However, these initial densities can control the dynamical transition from coccolithophores to diatoms because this transition is largely dependent on the kinetics of nutrient uptake of each individual population. In order to avoid this effect, our competition experiments were set up as invasion scenarios, such that the coccolithophore had roughly the same population density in all nutrient supply treatments and the coccolithophore to diatom (C/D) ratio was $\sim 8$ to 10 .

\section{RESULTS}

\section{Steady-state vs. non-steady-state nutrient supply dynamics}

Analyses of the uptake kinetics for nitrate in monospecific cultures of Thalassiosira pseudonana and Coccolithus braarudii grown at steady-state yielded maximum uptake rates and half saturation constants $( \pm$ $\mathrm{SE}$ ) of $2.18 \times 10^{-4} \pm 0.21 \times 10^{-4} \mu \mathrm{mol} \mathrm{cell}{ }^{-1} \mathrm{~d}^{-1}$ and $3.75 \pm$ $1.1 \mu \mathrm{mol} \mathrm{l}^{-1}$, respectively, for the diatom and $1.38 \times 10^{-4}$ $\pm 0.18 \times 10^{-4} \mu \mathrm{mol} \mathrm{cell}{ }^{-1} \mathrm{~d}^{-1}$ and $1.06 \pm 0.5 \mu \mathrm{mol} \mathrm{l}^{-1}$ for the coccolithophorid (Fig. 1A). A $t$-test comparing nonlinear regressions showed that the differences in the best-fit values of maximum nitrate uptake rates were statistically significant ( $t$-test, $t_{44}=2.89, \mathrm{p}<0.01$ ). Differences in half-saturation constants were also significant ( $t$-test, $\left.t_{44}=2.29, \mathrm{p}<0.05\right)$. Using these nutrient uptake kinetic parameters as reference, we conducted a simulation to examine the variability in $R^{*}$, the competition parameter, for each of our model species by assigning different potential values to their maximum growth rate and minimum and maximum cell quotas (Fig. 1B). We assumed increased nutrient requirements at higher maximum growth rate (Aksnes \& Egge 1991), which is a reasonable assumption as fast growing species typically possess higher minimum quotas and lower algal yields. The mortality rate was $0.25 \mathrm{~d}^{-1}$ (i.e. the dilution rate). The analysis suggested that the 
A

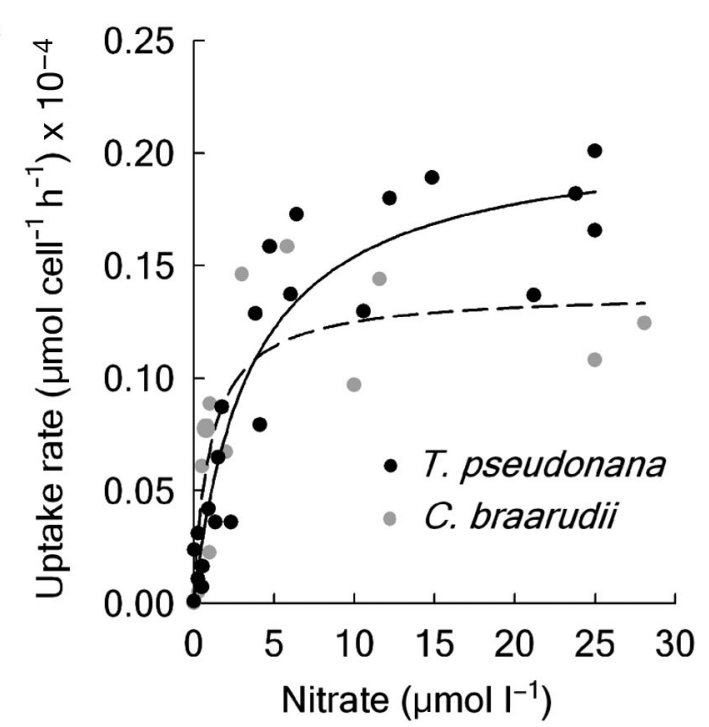

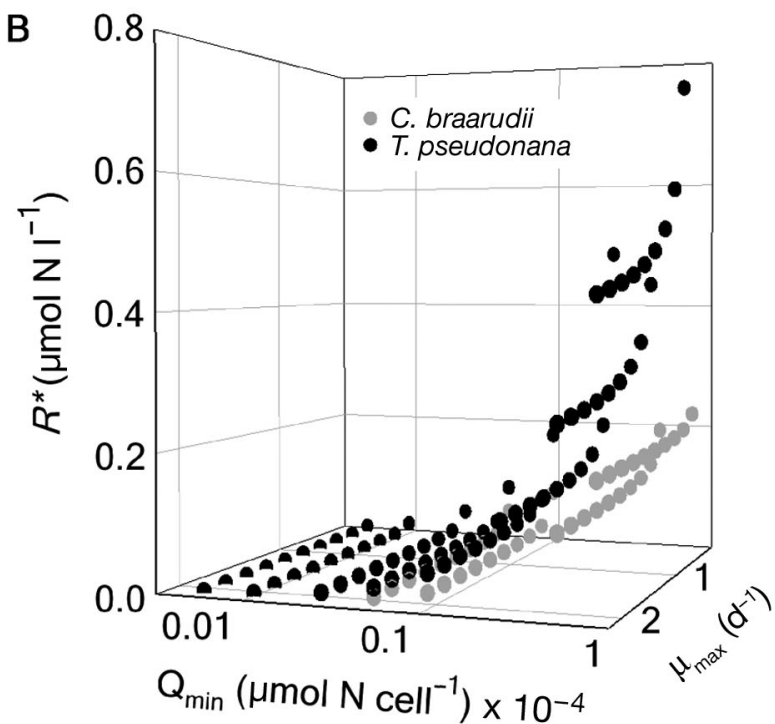

Fig. 1. Thalassiosira pseudonana and Coccolithus braarudii. (A) Nutrient uptake kinetics for the diatom T. pseudonana and the coccolithophore $C$. braarudii. The data are combined from 2 independent experiments on cells that had been acclimated in nitrate limited chemostats for a week. (B) Sensitivity of the competition parameter $\left(R^{*}\right)$ to simulated values of maximum growth rate $\left(\mu_{\max }\right)$ and cell quotas $\left(Q_{\min }\right)$. In all cases, the maximum cell quotas were assumed to be one-order of magnitude higher than the minimum cell quotas

coccolithophore, which had a lower $R^{*}$ for the whole range of assigned values, would outcompete the diatom under continuous nitrate limitation. For example, assuming a minimum quota of $0.5 \times 10^{-4} \mu \mathrm{mol}$ cell ${ }^{-1}$ and a maximum growth rate of $1.5 \mathrm{~d}^{-1}$, the model predicted an $R^{*}$ of 0.3 for the diatom and of 0.1 for the coccolithophore.

We initially introduced the 2 species at equal population densities in 2 independent cultures operating in parallel under similar temperature, light and nutrient supply regimes and began diluting with the inflow media. In both experiments, the nitrate in the outflow media became undetectable at $\sim$ Day 14 , and the data shown in Fig. 2 begin from this period, when the 2 species were in approximately equal abundance and the system had been operating in a steady-state mode (i.e. continuous nitrate limitation). According to Eq. (6) each population will reduce nitrate concentration in the bulk media to levels predicted by their $R^{*}$, and thus the species with the lowest requirements for nitrate should competitively displace the other. Consistent with this, continuous nitrate limitation in our experimental microcosms (i.e. the classical chemostat-like system) led to a rapid decrease in the abundance of Thalassiosira pseudonana and a simultaneous increase of Coccolithus braarudii (Fig. 2). The pattern was characterized by a progressive increase of the C/D ratio at a rate of $0.05 \log$ units $\mathrm{d}^{-1} . F_{\mathrm{v}} / F_{\mathrm{m}}$ ranged from $\sim 0.35$ to 0.55 (Fig. 3). Despite an elevated silicate to nitrate ratio of the inflow media $(>1.2)$, diatoms were excluded by the coccolithophore population under continuous nitrate limitation, which indicated that the availability of silicate per se is a necessary but insufficient condition for the increased population density of the diatom.

To avoid competitive exclusion of Thalassiosira pseudonana, steady-state nitrate supply conditions were maintained until the abundance of coccolithophorids exceeded $\sim 10$-fold the density of the diatom ( Day 22$)$. Thereafter, population dynamics were altered by providing pulses of nitrate ( $7 \mu \mathrm{mol} \mathrm{l}^{-1}$ twice per day). Assuming minimum intracellular nitrate quotas within the range of values used in our simulation analysis and equal resource partitioning (though unrealistic, this assumption is used for heuristic purposes), each nitrate pulse would have doubled or tripled cell quota. Indeed, deviation from steady-state nitrate supply rapidly switched the outcome of competitive interactions, allowing diatoms to dominate the assemblage (Fig. 2). $F_{\mathrm{v}} / F_{\mathrm{m}}$ increased to values above 0.5 (Fig. 3). Although initially nitrate pulses increased the density of both the diatom and coccolithophore populations, the C/D ratio showed a sharp and synchronous decrease, which highlights the competitive ability of the diatom under pulsed nutrient conditions (i.e. the population abundance of T. pseudonana increased much faster than that of Coccolithus braarudii) (Fig. 2). While nitrate was undetectable in the outflow media, phosphate and silicate were always in excess (Fig. 2). By Day 50, the C/D ratio fell to values as low as 0.2 , but the assemblage was still photosynthetically active, as indicated by $F_{\mathrm{v}} / F_{\mathrm{m}}$ values of $\sim 0.4$ (Fig. 3). These results were consistent in 2 independent cultures. 

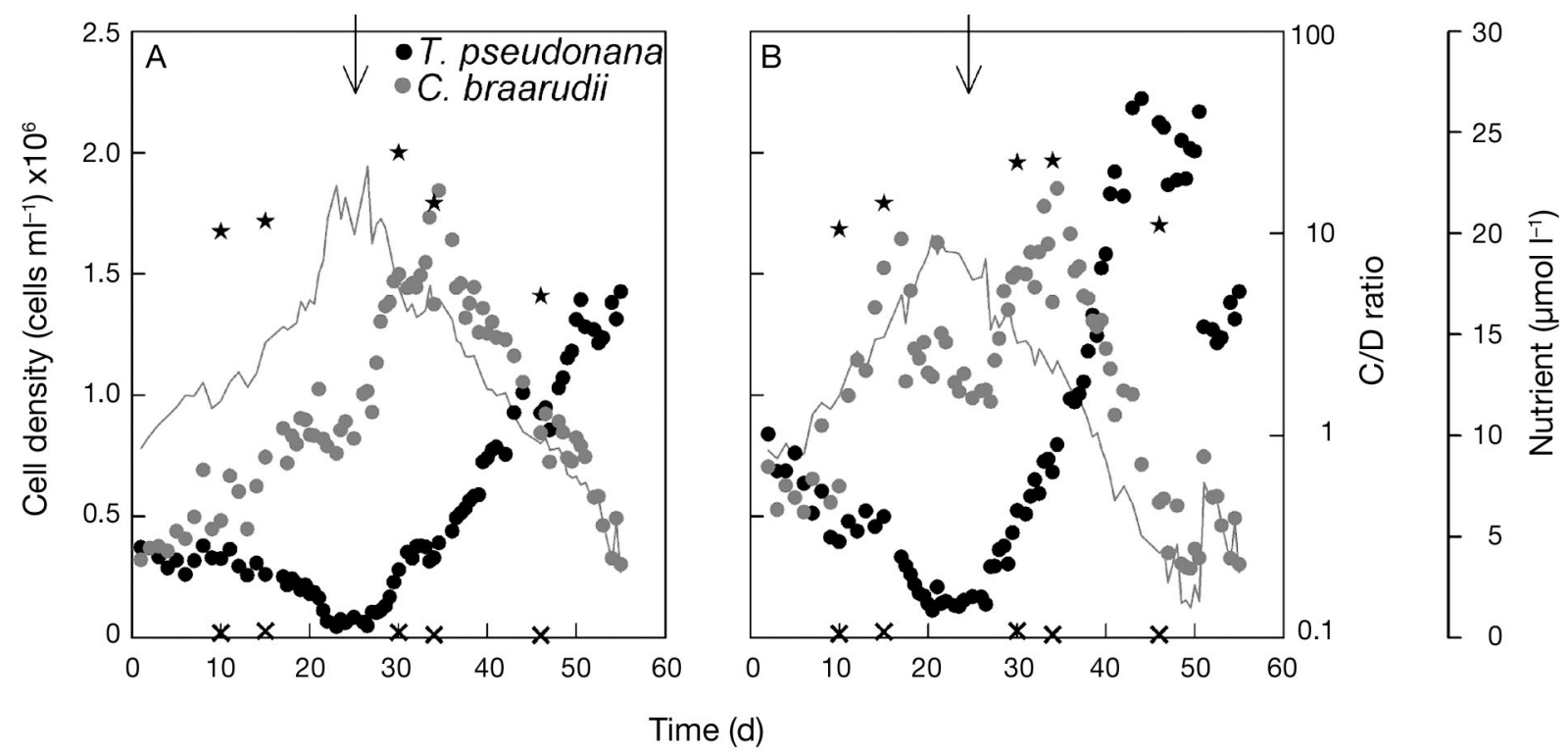

Time (d)

Fig. 2. Thalassiosira pseudonana and Coccolithus braarudii. Population dynamics of T. pseudonana (•) and C. braarudii $(\bullet)$ competing under nitrate-limiting and nitrate-pulsing conditions. The grey line represents the coccolithophores:diatoms (C/D) ratio on a logarithmic scale. (A) and (B) correspond to 2 experiments conducted independently of each other in parallel under similar nutrient, light and temperature regimes (true replicates). Nitrate concentrations on the outflow media $(x)$ were below detection throughout the experimental time shown, whereas silicate concentrations were by far in excess ( $\star$ ). The arrows mark the beginning of the nutrient pulsing $\left(2\right.$ pulses $\left.\mathrm{d}^{-1}\right)$ on Day 22

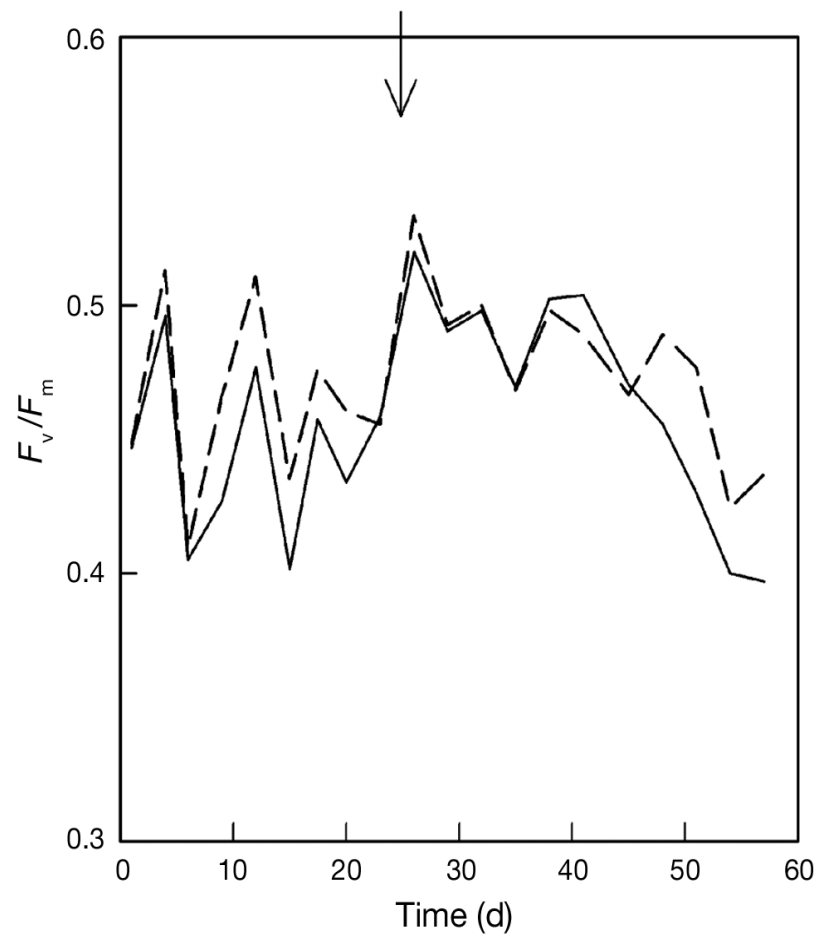

Fig. 3. Temporal changes in variable/maximum fluorescence $\left(F_{\mathrm{v}} / F_{\mathrm{m}}\right)$ of mixed culture assemblages. The temporal evolution of $F_{\mathrm{v}} / F_{\mathrm{m}}$ was averaged every $3 \mathrm{~d}$ for the experiments plotted in Fig. 2A (solid line) and Fig. 2B (dashed line). The arrow marks the beginning of the nutrient pulsing $\left(2\right.$ pulses $\left.\mathrm{d}^{-1}\right)$ on Day 22

\section{Quantifying the rate of competitive exclusion}

In most aquatic ecosystems, nutrient supplies are not constant over long periods of time and under certain circumstances (e.g. storms or internal waves) nutrientlimited phytoplankton can be transiently supplied with pulses of relatively high nutrient concentrations. In our experiment, we successfully manipulated selection of a coccolithophore and a diatom by simply adjusting the dynamics of nutrient supply (Fig. 2). However, the extent to which the dynamics and magnitude of nitrate inputs control the rate of competitive exclusion remained unknown. To explore this question, we conducted a suite of competition experiments in which the inflow media was supplemented with pulses of nitrate at 3 pre-determined time intervals: 0.5, 1 and 5 pulses $\mathrm{d}^{-1}$. These perturbation frequencies were chosen to simulate typical oceanographic events, such as upwelling pulses, internal or tidal waves and vigorous mixing associated with transient storms, phenomena which appear to alter population dynamics and community composition in nature (Walsh et al. 1978).

Competition experiments were set up as invasion scenarios (Tilman \& Sterner 1984). Initial population densities of the coccolithophore, the resident species, were $~ 8$-fold higher than those of the diatom, the invader species (Fig. 4). Nitrate pulses increased the abundance of diatoms at the expense of the cocco- 

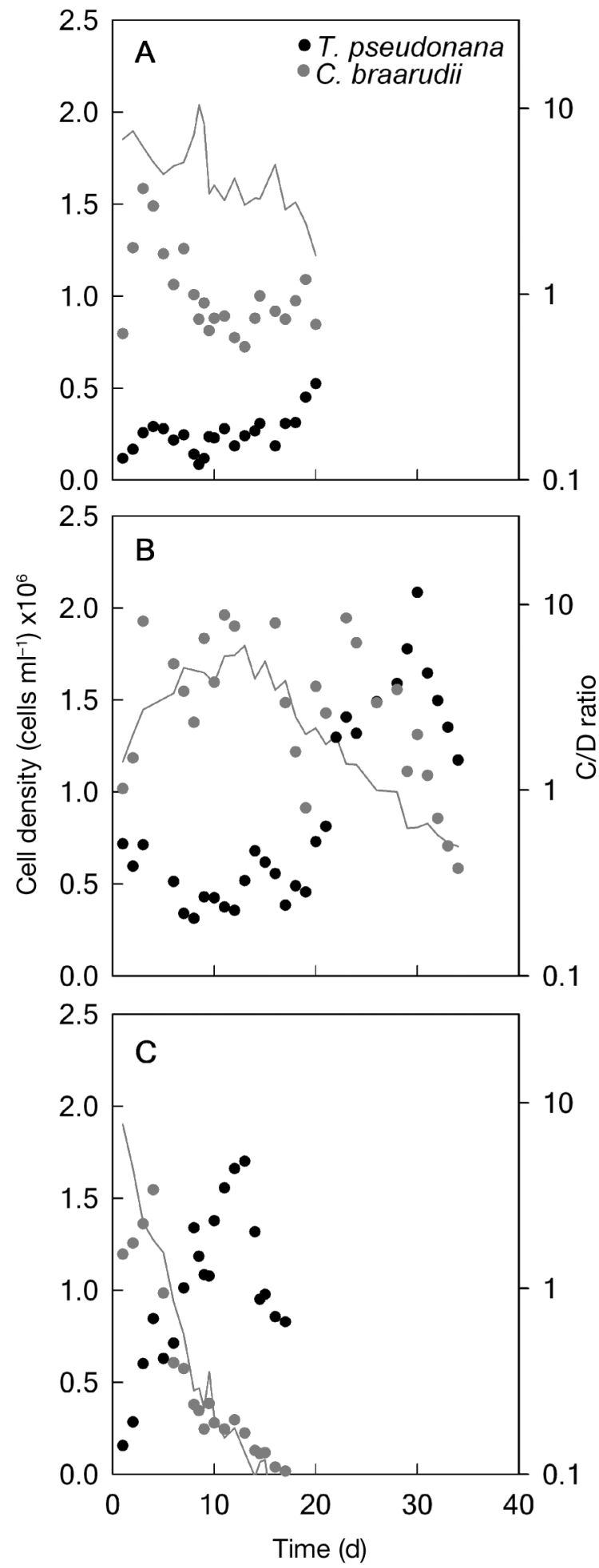

Fig. 4. Thalassiosira pseudonana and Coccolithus braarudii. Population dynamics of $T$. pseudonana $(\bullet)$ and $C$. braarudii $(\bullet)$ and the coccolithophore:diatom (C/D) ratio (grey line) at frequencies of (A) 0.5 , (B) 1 and (C) 5 nitrate pulses $\mathrm{d}^{-1}$. These experiments were conducted in chemostat systems by adding pulses of nitrate at predetermined time intervals. C/D ratio is shown on a logarithmic scale lithophore (i.e. the C/D ratio progressively declined over time), yet their population dynamics were markedly different among treatments (Fig. 4). Whereas the C/D ratio slightly decreased at 0.5 pulses of nitrate $\mathrm{d}^{-1}$, the rate of coccolithophore exclusion increased rapidly with the frequency of pulses and nitrate load (Fig. 5A). From the time series of population densities (Figs. $2 \& 4$ ), we calculated the rate of competitive exclusion. The response variable, C/D ratio $(t)=N 1(t) / N 2(t)$, where $N 1$ and $N 2$ are the densities of Coccolithus braarudii and Thalassiosira pseudonana, respectively, on day $t$, was regressed on a logarithmic scale against $t$. Then, the rate of competitive exclusion was calculated as the slope of the regression model between the $\log \mathrm{C} / \mathrm{D}$ ratio and time (Fig. 5A). Regardless of the perturbation frequency, T. pseudonana competitively displaced $C$. braarudii. The temporal decay of the $\log \mathrm{C} / \mathrm{D}$ ratio conformed consistently to a simple linear regression model (Fig. 5A). Plotting all treatments together, the analysis revealed that the rate of exclusion of $C$. braarudii increased linearly with the availability of nitrate (Fig. 5B). Higher frequencies of nitrate input accelerated exclusion, but only under an extreme condition of 5 pulses $\mathrm{d}^{-1}$ was $C$. braarudii completely excluded by Day 18. In the other treatments, the $\mathrm{C} / \mathrm{D}$ ratio showed a progressive decay, while the slower rate of competitive displacement precluded reaching complete exclusion of the coccolithophore over the time course of the experiments ( Day 30). Interestingly, including rates of exclusion of T. pseudonana under continuous nitrate limitation (i.e. derived from grey lines on Fig. 5A), the dataset conformed to a non-linear model that predicts coexistence at some range between $>0$ and 0.5 pulses $\mathrm{d}^{-1}$.

\section{DISCUSSION}

Our experimental results strongly suggest that the temporal supply of a single limiting nutrient can control the relative population densities of 2 species of phytoplankton. Built upon steady-state relationships, theoretical models and experimental analyses of resource competition have demonstrated that (1) among species competing for a single limiting resource only one species can persist, (2) species limited by different resources can coexist at equilibrium, and (3) the relative abundance of coexisting species is regulated by the ratio of limiting resources (Tilman 1982). For decades, these competition models have provided a fundamental basis to link algal physiology and community ecology, yet, are based upon the unrealistic assumption of environmental homogeneity.

Non-equilibrium competition theories have significantly improved our understanding of community 

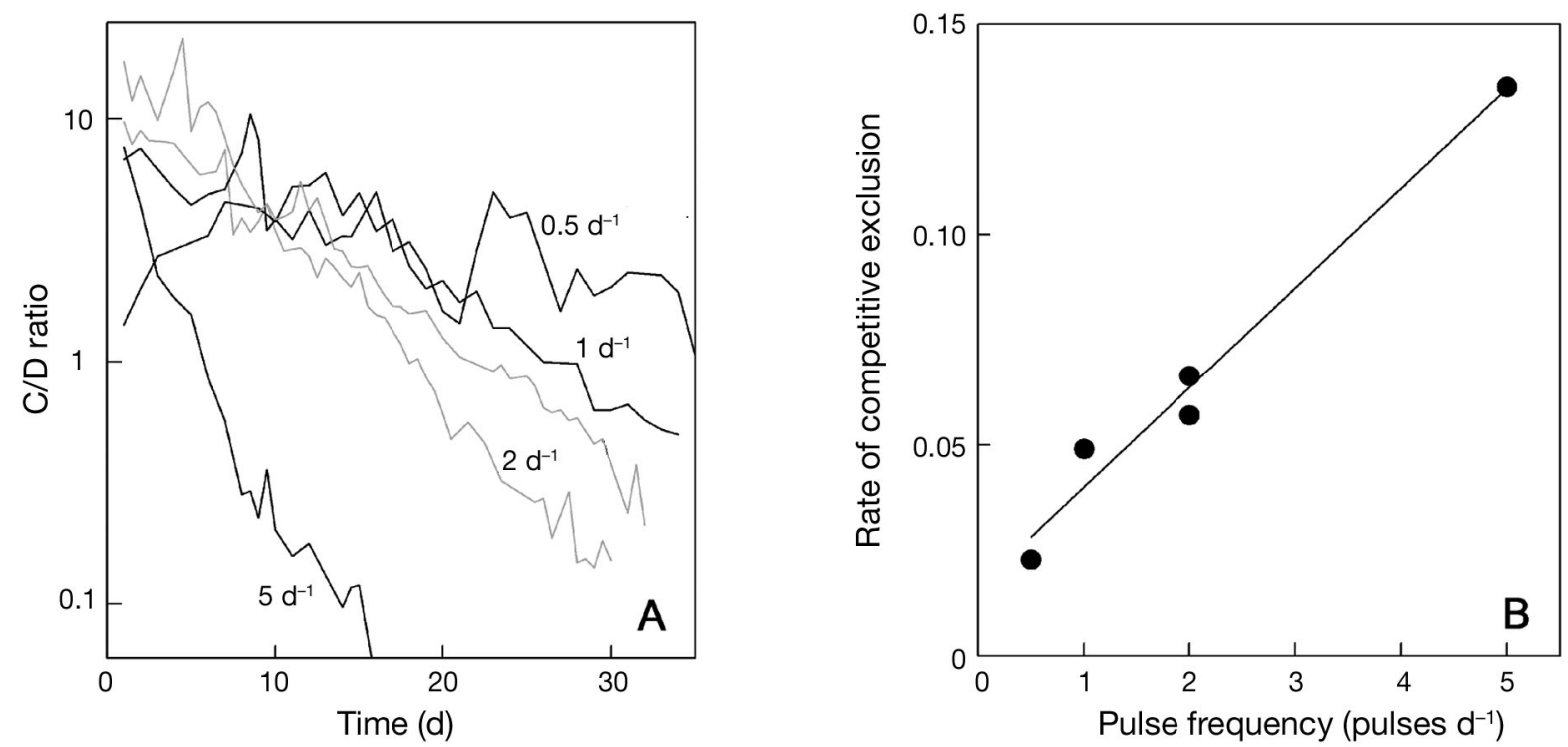

Fig. 5. (A) Changes in the coccolithophore:diatom (C/D) ratio over time at different frequencies of nitrate pulses. The temporal evolution of the C/D ratio from experiments shown in Fig. 2 (gray lines) and Fig. 4 (black lines) is shown on a logarithmic scale. The slope of the linear relationship between log C/D ratio and time represents the rate of competitive exclusion. For the 1 pulse $\mathrm{d}^{-1}$ treatment, the slope was calculated from the time at which the C/D ratio began to decrease. (B) Relationship between the rate of competitive exclusion and the rate of nitrate supply (frequency of nitrate pulses). The rate of competitive exclusion, calculated from the linear regression model of $\log \mathrm{C} / \mathrm{D}$ ratio over time, is negative and was multiplied by -1 for this illustration. The data fit to a linear regression model: $y=0.017+0.024 x, \mathrm{R}^{2}=0.97, \mathrm{p}=0.002$

dynamics (and biodiversity) by incorporating parameterizations that reproduce the variable efficiency of resource consumers in response to environmental stochasticity (Richerson et al. 1970, Grover 1991b). For instance, the observation that microbial plankton communities harbor more species than limiting resources, the so-called 'Paradox of the Plankton' (Hutchinson 1961), has been explained by the non-linearity of resource consumers in an ever changing scenario. The extent to which marine phytoplankton at cell densities typical of natural systems physically interact with each other is controversial (Siegel 1998). However, temporal variations in resource supply can alter the growth rate, resulting in niche segregation over time and the ensuing potential of low-density species to temporarily increase their population size. These non-equilibrium models provide a theoretical solution for the coexistence of species competing for the same limiting resource (Grover 1991b, Sommer 1993) and therefore offer a more realistic framework to approach the study of biotic interactions and community dynamics.

To the extent that microcosm experiments can inform us about natural aquatic ecosystems, we suggest that competition between marine diatoms and coccolithophores is particularly relevant to ocean biology and biogeochemistry (Smetacek 1999, Falkowski 2004). We were unable to experimentally simulate conditions that promote long-term coexistence of both populations. Either at equilibrium or under nutrient pulsing conditions, our laboratory-simulated ecosystem deviated towards the dominance of the coccolithophore or the diatom, respectively. However, we found an increasing linear relationship between the frequency of nitrate pulses and the rate of competitive exclusion, implying a longer coexistence at low perturbation frequencies. Consistent with our analysis of data, earlier competition experiments on green algae and photosynthetic prokaryotes have shown an increased rate of competitive exclusion with decreasing pulse period (Grover 1991a, Ducobu et al. 1998). These results indicate that nutrient supply dynamics can alter the strength of competitive interactions and hence control the temporal progression of the ecological succession and domain shifts of phytoplankton populations in the ocean.

The classical succession from diatom dominance to the abundance of non-siliceous taxa (or vice versa) traditionally has been explained as a result of changes in the ratio between the supply of silicate, an absolute requirement for diatoms, and other essential nutrients such as nitrogen, phosphorous or iron. While that chemical supply scenario may also lead to phytoplankton succession, our experimental results strongly suggest that it can also be a predictable manifestation of physically-forced variable nutrient supply regimes. An alternative possibility is that allelopathic effects, the production of substances that inhibit growth of neighboring species, could have influenced population 
dynamics in our experimental microcosms in a manner similar to that predicted by nutrient competition theory. However, though allelopathic interactions may contribute to regulating population dynamics, such an endogenous control is unlikely to influence the dynamical succession and domain shifts of phytoplankton species in the ocean.

The hypothesized role of nutrient supply dynamics in delaying, diverting or resetting phytoplankton succession, phenologies and domain shifts can also be viewed within the framework of the classical Connell's intermediate disturbance hypothesis (Connell 1978, Sommer 1995). Low disturbance frequencies promote competitive exclusion, while high disturbance selects for a few species tolerant to stress. Ocean stratification and the ensuing reduction of nutrient supply during, for instance, upwelling relaxation or the spring-summer transition in temperate seas would favor the dominance of taxa that take up nutrients slowly, such as coccolithophores or autotrophic prokaryotes. Conversely, high disturbance frequencies characteristic of coastal upwelling systems or open ocean regions with high submesoscale activity would select for a few 'opportunistic' groups such as diatoms that are adapted to such stressful conditions. These 2 extreme scenarios serve to illustrate the role of nutrient input dynamics in controlling the temporal progression of phytoplankton succession through an array of intermediate conditions. Although we emphasize here the importance of bottom-up controls, a number of mechanisms including predation, allelopathic interactions, dispersal, light intensity or temperature may further influence the dynamics of phytoplankton communities in nature (Reynolds 2006, Strom 2008).

The proposition that variable nutrient supplies can dominate the dynamics of phytoplankton communities implies the need for experimental approaches and modeling analyses at high spatial resolutions. Much evidence suggests the importance of vertical flux of mass associated with ocean fluid dynamics at scales of dozens of meters to hundreds of kilometers (sub- and mesoscale features) (Mahadevan et al. 2010, d'Ovidio et al. 2010). These oceanic features can play an important role in controlling community dynamics and shaping global patterns of phytoplankton distributions. Ocean biology models traditionally have given a larger importance to the effect of nutrient ratios, which can be easily inferred from property plots and isopycnal mixing lines. Instead, assessing the importance of variable nutrient supplies in ocean biology and biogeochemistry requires an understanding of mesoscale and submesoscale physical processes, which presents a new challenge for ocean biology modelers because these processes are not fully resolved in general circulation models at present.
The resource-ratio (chemical forcing) and the variable nutrient supply (physical forcing) theories offer alternative, though not mutually exclusive mechanisms, to explain phytoplankton succession and domain shifts in the ocean. Whereas the resource ratio theory is compatible with deviations of the nutrient supply ratios from the classical Redfield elemental stoichiometry, the variable nutrient supply theory conceptualized by Margalef is compatible with a temporal version of the Connell's intermediate disturbance hypothesis in which community dynamics rapidly respond to variations in the frequency of perturbations. Contemporaneous trends in climate warming may potentially alter upper ocean physics and nutrient supply regimes over different temporal and spatial scales giving rise to variations in the patterns of phytoplankton succession, phenologies and domain shifts.

Acknowledgements. We thank R. Lauck for nutrient analyses, and K. Richardson and 3 anonymous reviewers for comments on the manuscript. The research was supported by Plankton Tech. J-B.L. was supported by the Jeju Sea Grant College Program of Jeju National University, which is funded by the Korean Government. P.C. was supported by a Marie Curie International Fellowship within the 6th European Community Framework Program.

\section{LITERATURE CITED}

Archer D, Winguth A, Lea D, Mahowald N (2000) What caused the glacial/interglacial atmospheric $\mathrm{pCO}_{2}$ cycles? Rev Geophys 38:159-189

Aksnes DL, Egge JK (1991) A theoretical model for nutrient uptake in phytoplankton. Mar Ecol Prog Ser 70:65-72

> Boyd PW, Doney SC (2002) Modelling regional responses by marine pelagic ecosystems to global climate change. Geophys Res Lett 29, 1806, doi:10.1029/2001GL014130

Cermeño P, Dutkiewciz SW, Harris RP, Follows MJ, Schofield O, Falkowski PG (2008) The role of nutricline depth in regulating the ocean carbon cycle. Proc Natl Acad Sci USA 105:20344-20349

> Connell JH (1978) Diversity in tropical rain forests and coral reefs. Science 199:1302-1310

> d'Ovidio F, De Monte S, Alvain S, Dandonneau Y, Lévy M (2010) Fluid dynamical niches of phytoplankton types. Proc Natl Acad Sci USA 107:18366-18370

Droop MR (1973) Some thoughts on nutrient limitation in algae. J Phycol 9:264-272

Ducobu H, Huisman J, Jonker RR, Mur LR (1998) Competition between a prochlorophyte and a cyanobacterium under various phosphorus regimes: comparison with the Droop model. J Phycol 34:467-476

> Egge JK, Aksnes DL (1992) Silicate as regulating nutrient in phytoplankton competition. Mar Ecol Prog Ser 83:281-289

Falkowski PG (2002) The ocean's invisible forest-Marine phytoplankton play a critical role in regulating the earth's climate. Could they also be used to combat global warming? Sci Am 287:54-61

Falkowski PG (2004) The evolution of modern eukaryotic phytoplankton. Science 305:354-360

Falkowski PG, Oliver M (2007) Mix and match: how climate 
selects phytoplankton. Nat Rev Microbiol 5:813-819

Falkowski PG, Raven JA (1997) Aquatic photosynthesis. Blackwell Scientific Publishers, Oxford

Falkowski PG, Laws EA, Barber RT, Murray JW (2003) Phytoplankton and their role in primary, new, and export production. In: Fasham MJR (ed) Ocean biogeochemistry: the role of the ocean carbon cycle in global change. Global Change -The IGBP Series. Springer, Berlin, p 99-122

Gorbunov MY, Kolber ZS, Falkowski PG (1999) Measuring photosynthetic parameters in individual algal cells by Fast Repetition Rate fluorometry. Photosynth Res 62: 141-153

Grasshoff K (1976) Methods of seawater analysis. Verlag Chemie, New York, NY

Grover JP (1988) Dynamics of competition in a variable environment: experiments with two diatom species. Ecology 69:408-417

Grover JP (1989) Effects of Si:P supply ratio, supply variability, and selective grazing in the plankton: an experiment with a natural algal and protistan assemblage. Limnol Oceanogr 34:349-367

Grover JP (1990) Resource competition in a variable environment: phytoplankton growing according to Monod's model. Am Nat 136:771-789

Grover JP (1991a) Dynamics of competition among microalgae in variable environments: experimental tests of alternative models. Oikos 62:231-243

Grover JP (1991b) Resource competition in a variable environment: phytoplankton growing according to the variable-internal-stores model. Am Nat 138:811-835

Holligan PM, Robertson JE (1996) Significance of ocean carbonate budgets for the global carbon cycle. Glob Change Biol 2:85-95

Hood RR, Lawson EA, Armstrong RA, Bates NR and others (2006) Pelagic functional group modeling: progress, challenges and prospects. Deep-Sea Res II 53:459-512

Hutchinson GE (1961) The paradox of the plankton. Am Nat 95:137-145

> Iglesias-Rodríguez MD, Brown CW, Doney SC, Kleypas J and others (2002) Representing key phytoplankton functional groups in ocean carbon cycle models: Coccolithophorids. Global Biogeochem Cycles 16, 1100, doi:10.1029/2001GB001454

Legoviç T, Cruzado A (1997) A model of phytoplankton growth on multiple nutrients based on the MichaelisMenten-Monod uptake, Droop's growth and Liebig's law. Ecol Model 99:19-31

> Litchman E, Klausmeier CA, Schofield O, Falkowski PG (2007) The role of functional traits and trade-offs in structuring phytoplankton communities: scaling from cellular to ecosystem level. Ecol Lett 10:1170-1181

Mahadevan A, Tandon A, Ferrari R (2010) Rapid changes in mixed layer stratification driven by submesoscale instabilities and winds. J Geophys Res 115, C03017, doi:10.1029/ 2008JC005203

Margalef R (1978) Life-forms of phytoplankton as survival alternatives in an unstable environment. Oceanol Acta 1:493-509

Miller TE, Burns JH, Munguia P, Walters EL and others (2005) A critical review of twenty years' use of the resource-ratio theory. Am Nat 165:439-448

Raven JA (1997) The vacuole: a cost benefit analysis. In: Leigh RA, Sanders, D, Callow JA (eds) Advances in Botanical Research, Vol 25. Academic Press, London, p 59-86

Reynolds CS (2006) The ecology of phytoplankton. Cambridge University Press, New York, NY

Richerson P, Armstrong R, Goldman CR (1970) Contempora- neous disequilibrium, a new hypothesis to explain the 'Paradox of the Plankton'. Proc Natl Acad Sci USA 67: 1710-1714

> Siegel D (1998) Resource competition in a discrete environment: why are plankton distributions paradoxical? Limnol Oceanogr 43:1133-1146

> Sieracki ME, Verity PG, Stoecker DK (1993) Plankton community response to sequential silicate and nitrate depletion during the 1989 North Atlantic spring bloom. Deep-Sea Res II 40:213-225

Smayda TJ (1989) Primary production and the global epidemic of phytoplankton blooms in the sea: a linkage? In: Cosper EM, Bricelj VM, Carpenter EJ (eds) Novel phytoplankton blooms. Springer-Verlag, Berlin, p 449-483

Smayda TJ, Reynolds CS (2001) Community assembly in marine phytoplankton: application of recent models to harmful dinoflagellate blooms. J Plankton Res 23:447-461

Smetacek V (1999) Diatoms and the ocean carbon cycle. Protist 150:25-32

Sommer U (1983) Nutrient competition between phytoplankton species in multispecies chemostat experiments. Arch Hydrobiol 96:399-416

> Sommer U (1985) Comparison between steady state and nonsteady state competition: experiments with natural phytoplankton. Limnol Oceanogr 30:335-346

Sommer U (1993) Phytoplankton competition in Plußsee: a field test of the resource-ratio hypothesis. Limnol Oceanogr 38:838-845

> Sommer U (1995) An experimental test of the intermediate disturbance hypothesis using cultures of marine phytoplankton. Limnol Oceanogr 40:1271-1277

> Stewart FM, Levin BR (1973) Partitioning of resources and the outcome of interspecific competition: a model and some general considerations. Am Nat 107:171-198

Stolte W, Riegman R (1995) Effect of phytoplankton cell size on transient-state nitrate and ammonium uptake kinetics. Microbiology 141:1221-1229

Strom SL (2008) Microbial ecology of ocean biogeochemistry: a community perspective. Science 320:1043-1045

Tilman D (1977) Resource competition between plankton algae: an experimental and theoretical approach. Ecology 58:338-348

Tilman D (1982) Resource competition and community structure. Princeton University Press, Princeton, NJ

Tilman D, Sterner RW (1984) Invasions of equilibria: tests of resource competition using two species of algae. Oecologia 61:197-200

Tilman D, Kilham SS, Kilham P (1982) Phytoplankton community ecology: the role of limiting nutrients. Annu Rev Ecol Syst 13:349-372

Tozzi S, Schofield O, Falkowski P (2004) Historical climate change and ocean turbulence as selective agents for two key phytoplankton functional groups. Mar Ecol Prog Ser 274:123-132

- Tréguer P, Pondaven P (2000) Global change: silica control of carbon dioxide. Nature 406:358-359

Volk T, Hoffert MI (1985) Ocean carbon pumps: analysis of relative strengths and efficiencies in ocean-driven atmospheric $\mathrm{CO}_{2}$ changes. In: Sundquist ET, Broecker WS (eds) The carbon cycle and atmospheric $\mathrm{CO}_{2}$ : natural variations Archean to present. Geophysical Monograph, Vol. 32, American Geophysical Union, Washington DC, p 99-110

> Walsh JJ, Whitledge TE, Barvenic FW, Wirick CD, Howe SO, Esaias WE, Scott JT (1978) Wind events and food chain dynamics within the New York Bight. Limnol Oceanogr 23:659-683

Submitted: June 14, 2010; Accepted: February 15, 2011

Proofs received from author(s): May 9, 2011 